\title{
Extending the concept of pro-environmental action and behaviour: a binary perspective
}

\begin{abstract}
Many studies have explored pro-environmental actions. This paper aims to extend the concept of pro-environmental action in the context of binary themes or perspectives. The extended conceptualisation is drawn from a study that investigates the influence of residential outdoor environmental education courses on Malaysian participants' environmental attitudes and behaviours using a life history approach. Data analysis revealed six binary pro-environmental actions: conforming/transforming action, compulsory/optional action, intentional/unintentional action, public/private sphere action, activist/non-activist action, and biospheric/anthropocentricoriented action. The binary actions presented in this paper pull together all of the binary themes, both pre-existing ones and those emerging from the current study data. The paper provides recommendations about how one can contribute to environmental quality and positively impact the sustainable development agenda.
\end{abstract}

Keyword: Outdoor education; Environmental education; Residential education; Proenvironmental action; Sustainable development 